difference between the largest diameter during the "imaging", period and the diameter in the last frame $(.5 \mathrm{sec})$ before stimulus presentation. The GSR change score was calculated by subtracting the maximum deflection (decrease in resistance) during the "imaging" period from the resting level during the last $1 / 2 \mathrm{sec}$ before presentation of the stimulus. ${ }^{2}$

Concrete and abstract nouns differed significantly in terms of mean pupillary change scores, with greater dilation $(\mathrm{t}=2.23, \mathrm{p}<.05)$ occurring to the abstract words (Fig. 1). The mean GSR change scores for concrete and abstract words did not differ significantly $(\mathrm{t}=1.12, \mathrm{p}<.10)$.

$$
\text { Response Latency }
$$

Key press, PR, and GSR latency scores were analyzed. Pupillary latency was the time to maximum dilation after stimulus presentation, and GSR latency was the time to maximum deflection. The mean key-press latencies for the two types of nouns differed significantly $(t=6.18, p<.001)$ with longer latencies occurring to the abstract nouns. Concrete and abstract nouns differed significantly in terms of mean pupillary latency, with longer latencies $(t=3.55, p<.01)$ occurring to the abstract nouns (Fig. 1). Significantly longer GSR latencies $(\mathrm{t}=2.49$, $\mathrm{p}<.05)$ also occurred to the abstract nouns. Correlations

The pupil and GSR change scores were converted to standard scores. A correlational analysis of mean scores over all stimuli for each $S$ revealed a significant $(\mathrm{df}=15, \mathrm{p}<.05)$ correlation of .50 between PR and GSR change scores and also a significant $(\mathrm{df}=15, \mathrm{p}<.05)$ correlation of .53 between PR magnitude and PR latency. Longer latencies were associated with larger pupil size.

\section{DISCUSSION}

The results support the conclusion that pupillary dilation is highly related to the "cognitive difficulty" of the imagery task. Both the magnitude and the latency of the pupillary response were significantly greater for abstract than for concrete words. These data are consistent with previous results (Paivio \& Simpson, 1968; Simpson, Molloy, Hale, \& Climan, 1968) in suggesting that pupillary latency may be more sensitive than magnitude as an indicator of cognitive task difficulty. The abstractness of the stimulus words accounts for $27 \%$ of the variance in PR latency scores while accounting for only $11 \%$ of the variance in PR magnitude scores.

The GSR does not seem to be as strongly related to degree of difficulty in image formation. The magnitude of the GSR did not significantly differentiate abstract from concrete nouns even though the GSR change score correlated with the PR change score. Kling \& Schlosberg (1961) reported results which suggest that GSR reflects attentional processes. These authors found increases in conductance when Ss were warned to get ready to work and again when warned to stop work and to rest. These responses would not habituate out and the authors concluded that each shift of attention from one phase to another of even a familiar task is marked by an increase in activation or arousal. This suggests that, in the imagery task, the GSR was reflecting shifts in attention from one word to the next. GSR latency, on the other hand, differed significantly for the two types of nouns, suggesting that, if GSR is reflecting an attentional process, it may somehow be related to the concrete-abstract dimension.

The results of the present investigation, then, suggest that pupillary dilation is more sensitive than the GSR to changes in cognitive activity occurring during an imagery task. In addition, they suggest that the latency of such physiological responses may be a more sensitive indicator than is the magnitude of these responses.

\section{REFERENCES}

BERRY, R. N. Skin conductance levels and verbal recall. Journal of Experimental Psychology, 1962a, 63, 275-277.

BERRY, R. N. Task difficulty, performance and skin conductance. Perceptual \& Motor Skills, $1962 \mathrm{~b}, 15,474-477$.

DAVIS, R. C. Modification of the galvanic reflex by daily repetition of a stimulus. Journal of Experimental Psychology, 1934, 17, 504-535.

HESS, E. H. Attitude and pupil size. Scientific American, 1965, 212, 46-54.

HESS, E.H., \& POLT, J. M. Pupil size in relation to mental activity during simple problem solving. Science, 1964, 143, 1190-1192.
KAHNEMAN, D., \& BEATTY, J. Pupil diameter and load on memory. Science, 1966, 154, 1583-1585.

KLING, J. W., \& SCHLOSBERG, H. Relation of skin conductance and rotary pursuit during extended practise. Perceptual \& Motor Skills, $1961,12,270-275$.

PAIVIO, A. Latency of verbal associations and imagery to noun stimuli as a function of abstractness and generality. Canadian Journal of Psychology, 1966, 20, 378-387.

PAIVIO, A., \& SIMPSON, H. M. The effect of word abstractness and pleasan tness on pupil size during an imagery task. Psychonomic Science, $1966,5,55-56$.

PAIVIO, A., \& SIMPSON, H. M. Magnitude and latency of the pupillary response during an imagery task as a function of stimulus abstractness and imagery ability. Psychonomic Science, 1968, 12, 45-46.

PAIVIO, A., YUILLE, J. C., \& MADIGAN, S. A. Concreteness, imagery, and meaningfulness values of 925 nouns. Journal of Experimental Psychology Monograph Supplement, 1968,76, No. 1.

SILVERMAN, S. J., COHEN, S. I., \& SCHMAVONIAN, B. M. Investigation of psychophysiologic relationships with skin resistance measures. Journal of Psychosomatic Research, 1959, 4, 65-87.

SIMPSON, H. M., MOLLOY, F. M., HALE, S. H. \& CLIMAN, M. H. Latency and magnitude of the pupillary response during an imagery task. Psychonomic Science, 1968, 13, 293-294.

YUILLE, J. C., \& PAIVIO, A. Latency of imaginal and verbal mediators as a function of stimulus and response concreteness-imagery. Journal of Experimental Psychology, 1967, 75, 540-544. NOTES

1. This research was supported by Grants APA-304 (to the first au thor) and APA-87 (to the second author) from the National Research Council of Canada, and a grant from the University of Western Ontario Research Fund.

2. No significant correlations were found between prestimulus level and maximum change scores for either PR or GSR.

\title{
Counterconditioning, neutral conditioning and extinction effects for the meaning of nonsense syllables
}

\section{ADAM MILLER, Saint Cloud State College, St. Cloud, Minn. 56301, and NORMA CLARK, University of Denver, Denver, Colo. 80210}

This study examined the effects of counterconditioning, neutral conditioning, and extinction, and different amounts of each, upon reshifting the rated meaning of nonsense syllables. For 120 Ss only counterconditioning was significantly effective at changing meaning.
Studies of the processes and conditions basic to change of word meaning have begun to identify a pattern among relationships. Using paired-associate learning procedures, the rated meaning of words, phrases, and nonsense syllables has been predictably changed. Designation of the change process as higher-order classical conditioning (Staats, 1968) has been accompanied by a series of studies delimiting the characteristics of the process and the variables related to it. One unexpected 
finding of Insko \& Oakes (1966), Miller \& Barsness (1969), and Miller et al (1969) is that extinction procedures do not change rated meaning of words and nonsense syllables. The experiment reported here focused upon three procedures for shifting rated meaning of nonsense syllables following an initial change.

\section{METHOD}

One hundred and twenty undergraduate volunteers were distributed among six groups of $20 \mathrm{Ss}$ each. For all Ss the five experimental steps were: preassociation rating, paired-associate learning, postassociation rating, change learning, and postchange rating. The rating sheet for the three rating steps included four nonsense syllables (NS) and 11 meaningful words (MW). Each was rated on the $\operatorname{good}(=1) \operatorname{bad}(=7)$ semantic differential scale. The NSs were XAD, XAZ, KUJ, and KUQ, selected for low norm-association values.

In paired-associate learning, one NS was projected on a screen for $10 \mathrm{sec}$ and $\mathrm{E}$ said a MW, which the group of Ss repeated aloud. The inter-NS interval was 1 sec. Each of the four NSs was shown 16 times within a random order of 64 presentations. XAD was paired with each of eight MWs of good meaning ( 1.30 to 1.57 on the good-bad scale), and KUJ was paired with each of eight MWs of bad meaning (6.37 to 6.67) based on the norms of Jenkins et al (1958). $\mathrm{XAZ}$ and $\mathrm{KUQ}$, similar in number of letters to the other two NSs, were presented without MW pairs. A memory test followed paired-associate learning.

In change learning, three different methods patterned on the above paired-associate procedure were used. For neutral conditioning, $\mathrm{XAD}$ and KUJ were paired with MWs of neutral norm-association values (4.50 to 5.50$)$ on the good-bad scale. This procedure was labeled extinction by Insko \& Oakes (1966). For counterconditioning XAD was paired with bad MWs and KUJ with good MWs, reversed from the original association. For extinction XAD and KUJ were presented without MW associates. For each change procedure the four NSs were shown and two amounts of change were used: 16 NS presentations, four of each NS, or $64 \mathrm{NS}$ presentations.

Thus, a 3 by 2 factorial design included three levels of change procedure and two levels of amount of change. The dependent variable was the ratings of NSs. The above procedures are assumed to represent classical conditioning: $\mathrm{XAD}$ and $\mathrm{KUJ}$ are CSs. The MWs of common meaning are UCSs, and their common meaning which is presumed to be a physiolgical reaction is an
UCR. The rating of NSs is mediated by the UCSs. Acquisition is shown following paired association by change in NS ratings toward the values of the MWs associated with the NS. Generalization occurs when a NS similar to the CS (XAZ similar to XAD and KUQ similar to KUJ) correspondingly changes in rated meaning following paired association of the CS. Extinction is demonstrated when, following presentation of NSs alone, the ratings return to neutral value. Since, in acquisition for this study, similar CSs were presented without UCS pairing, this procedure can be considered discrimination.

\section{RESULTS}

The effects of paired-associate learning were based on changes in mean ratings of KUJ and XAD from preassociation to postassociation ratings. A mean increase (+) indicated rating shifts toward bad meaning while a mean decrease (-) showed shift toward good meaning. KUJ changed from 4.24 to $5.27(+1.03)(t=5.15, d f=119$, $\mathrm{p}<.01)$. XAD changed from 4.58 to 2.77 $(-1.81)(\mathrm{t}=8.62, \mathrm{df}=119, \mathrm{p}<.01)$. Both changes were toward the values of the $M W$ associates and were consistent with the concept of acquisition for classical conditioning. The corresponding changes of the similar NSs, KUQ and XAZ, were +0.49 $(t=2.72, p<.05)$ and $-0.21 \quad(t=1.31$, n.s.), suggesting limited generalization. Since the acquisition process involved discrimination, no generalization should be expected.

Effects of three change procedures (counterconditioning, neutral conditioning, and extinction) and of two amounts of change learning (16 vs 64 presentations) were based on analyses of variance for postassociation and postchange ratings. The first analysis, for the NS KUJ, showed as significant sources of variance: change procedures $[F(2,114)=4.59, p<.05]$, rating times $[F(1,114)=23.30, p<.01]$, and their interaction $[F(2,114)=16.98$, $\mathrm{p}<.01]$. For counterconditioning the mean rating changes were from 5.40 to 3.13 $(-2.27)(t=5.54, d f=39, p<.01)$. The nonsignificant mean changes for neutral conditioning and extinction were, respectively, 5.20 to $4.83(-0.37)$ and 5.20 to $5.30(+0.10)$. For KUJ, the amount of change learning was not a significant source of variance.

The second analysis of variance for the NS XAD also showed as significant sources of variance: change procedure $[F(2,114)=10.73, p<.01]$, rating times $[F(1,114)=11.05, p<.01]$, and their interaction $[\mathrm{F}(2,114)=11.07, \mathrm{p}<.01]$. For counterconditioning, the mean rating change was from 3.00 to $4.75(+1.75)$ $(t=4.07, \quad d f=39, \quad p<.01)$. The corresponding changes for neutral conditioning and extinction were 2.80 to $3.03(+0.23)$ and 2.50 to $2.30(-0.20)$, both nonsignificant. For XAD, the amount of change learning was significant $[F(1,114)=5.61, p<.01]$, with the larger number of NS presentations producing the greatest rating change.

\section{DISCUSSION}

Acquisition, a frequently replicated finding, was demonstrated. The use of discrimination has not been previously reported. Extinction and neutral conditioning were ineffective for changing NS meaning, in accord with results of Insko \& Oakes (1966), Miller \& Barsness (1969), and Miller et al (1969), but in conflict with Das \& Mitra (1965). Generalization occurred to a limited degree in spite of discrimination conditioning. Limited generalization was also reported by Miller \& Barsness (1969). In contrast, Miller et al (1969) found no significant generalization even when they used simple acquisition rather than discrimination conditioning. The amount of counterconditioning was significant in affecting ratings of only one NS in this study, whereas Miller et al (1969) found no effect from amount of counterconditioning.

The accumulating evidence concerning the processes for changing rated meaning of MWs and NSs is revealing a process similar, but certainly not identical, to the modal characteristics of classical conditioning. But it is probable that no single kind of response fits the paradigm. One major problem in all recent studies of higher-order conditioning of word meaning is that the CR (a presumed physiological response) is indirectly measured through ratings. A direct measurement of the $C R$ is in order for studies such as the present one.

\section{REFERENCES}

DAS, J. P., \& MITRA, A. K. Persistence of acquired meaning in semantic conditioning. Psychonomic Science, 1965, 3, 83-84.

INSKO, C. A., \& OAKES, W.F. A wareness and the "conditioning" of attitudes. Journal of Personality \& Social Psychology, 1966, 4, 487-496.

JENKINS, J. J., RUSSELL, W. A., \& SUCI, G. J. An atlas of semantic profiles for 360 words. American Journal of Psychology, 1958, 71, 688-699.

MILLER, A. W., JR., \& BARSNESS, W. Extinction, stimulus generalization and partial reinforcement for higher-order meaning conditioning. Psychological Reports, 1969, 24, 288-290.

MILLER, A., GIMPL, M., \& MCCRIMMON, R. Extinction versus counterconditioning for the meaning of words and nonsense syllables. Psychonomic Science, 1969, 15, 92-93.

STAATS, A. W. Learning, language and cognition. New York: Holt, Rinehart \& Winston, 1968. 